\title{
Simulation for Analog Circuit Fault Propagation with Complex Networks
}

\author{
Meie Shen, Minfang Peng, Jianbiao He, and Kai Xie
}

\begin{abstract}
Based on complex network theory, the simulation method for analog circuit fault propagation behavior and its internal mechanism is proposed and the modeling approach for fault propagation networks is described. Taken the international standards circuit under test as an example, the network degree, clustering coefficient, average path length and other statistical parameters in the model are calculated. The small-world and scale-free properties of the analog circuit fault propagation network are verified. Therefore, efficient diagnosis information can be obtained from the constructed the network.
\end{abstract}

Index Terms-Analog circuit, fault propagation, simulation, Modeling, complex network.

\section{INTRODUCTION}

Even since 70's in last century, the fault diagnosis of analog circuit have been a hot research issue in the field of circuit system, and obtained many achievements in theory and method [1]-[6], but with the increase of analog circuit scale, complication and integration, there are three difficulties in the diagnosis and test task. One is the lack of a simple fault model, another is available diagnosis information insufficiency, and the third one is low robustness in fault identification and location. In this paper, we focus on the facts as follows. One hand, a tiny fault signal would be propagated, diffused, accumulated and amplified along with the topology of analog circuits, seriously affect the reliability and security of the circuits, and badly interfere with the fault location result; on the other hand, this abnormal signal is a significant information source of fault diagnosis. So, it is a key point in our research that how to effectively utilize the fault propagation property to mine deep diagnosis knowledge, complete the fault-tracing, and further more, propose some references for optimal design of analog circuit.

Manuscript received March 9, 2013; revised June 28, 2013. This work was supported by National Natural Science Foundation of China under Grants 61173108, 61272147, 60973032 and 60673084, and Hunan Provincial Natural Science Foundation of China under Grants 06JJ4075 and 10JJ2045.

Meie Shen is with the College of Computer Science, Beijing University of Information Science and Technology, Beijing,100101, China (e-mail: smelk@163.com)

Minfang Peng is with the College of Electrical and Information Engineering, Hunan University, Changsha, 410082, China (e-mail: pengminfang@ hnu.edu.cn).

Jianbiao He is with the School of Information Science \& Engineering, Central South Uinversity, Changsha, 410083, China, (e-mail: jbhe@ mail.csu.edu.cn)

Kai Xie is with the Electronics and Information School, Yangtze University, Hubei, 434023, China (e-mail: pami2009@163.com)
Compared to the previous modeling and analysis of analog circuit with complex networks, this paper's difference lies in the fact that its study does not focus on the network abstraction of the circuit physical topology, but places extra emphasis on the analysis and research on the network model endowed with statistical probability weight and fuzzy pattern division of the characteristics and behavior of the circuit such as fault propagation, which depends on the circuit structure and topology. From the view of fault diagnosis, obviously, our study is of not only greatly theoretical value to reveal the statistical properties of analog circuit fault feature space, but also important practical significance on deep fault diagnosis information mining. In this paper, various simulation methods and software have been used. By using the methods such as semi-quantitative analysis, it can effectively resolve the analog circuit components tolerance. In the simulation software, automated information exchange between circuit simulation and numerical calculation software makes it possible to carry on the co-simulation and statistical analysis of fault propagation behavior automatically and non-manualintervention in analog fault diagnosis simulation before test.

The paper is organized as follows: In Section II, we present the simulation frame and steps and the modeling method of fault propagation behavior along with analog circuits structure in detail. Together with a case study, a series of characteristics and parameters are obtained, the optimum proposal of accessible nodes is proposed and the fault propagation paths are acquired from the proposed model in Section III.

\section{Fault Propagation Network Modeling}

\section{A. Overview}

Based on complex network theory and data acquisition technology[7]-[16], by using co-simulation technology with Orcad, Matlab and Pajek, an analog circuit simulation model is established so as to acquire the potential information for performing fault propagation analysis. The block diagram in general on construction steps of the proposed fault propagation network model can be shown as Fig. 1, and divided into 3 parts (Orcad, Matlab and Pajek).

\section{Orcad:}

1) Completes a series of parameters analysis such as frequency response.

2) Obtains the qualitative and quantitative knowledge characterizing object properties.

3) Achieve the target of optimal choice for the initial simulation objects.

4) Extracts the change law of the parameters properties;

5) Provides evidence for sample points division of 
object data universe.

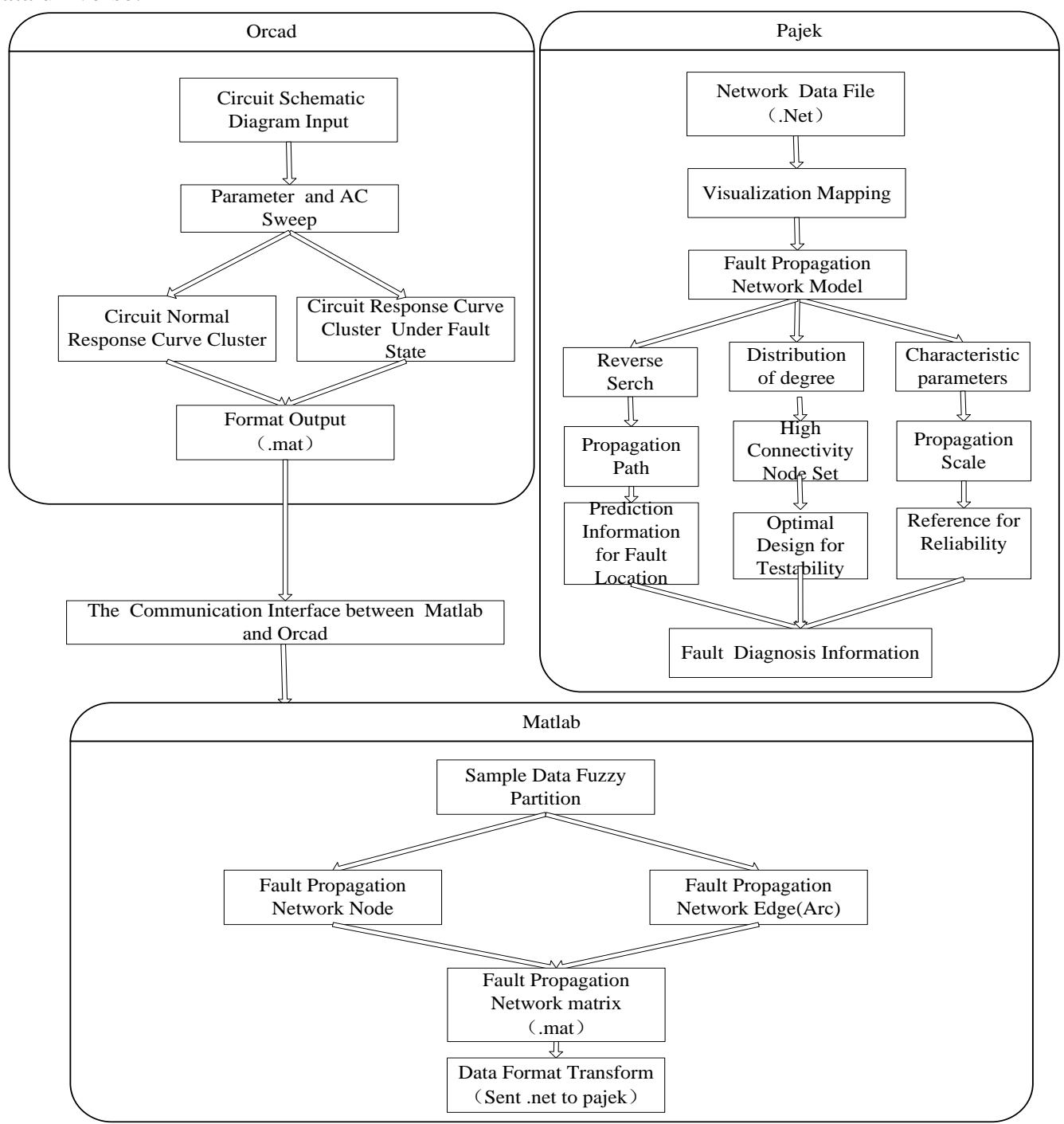

Fig. 1. Outline for the construction of the proposed fault propagation network model.

\section{Matlab:}

1) Realizes the organic synthesis of the experimental data and prior knowledge.

2) Completes the construction of fault propagation network model.

3) Describes some model characteristics such as fault propagation probability.

Pajek:

1) Realizes the visualization of the network mode.

2) Completes some parameters analysis including degree distribution,clustering coefficient, average path length and so on.

\section{B. Fault Feature Simulation}

According to severity, the fault in analog circuit can be divided into hard fault and soft fault. Hard fault had mutations in element parameters, which mainly includes open-circuit fault and short-circuit fault. Soft fault shows an element parameters deviation with time and environment to an unallowable extent. In analog circuit, element parameters maybe change continuously on $[0,+\infty)$, and different parameters-variable have different degree of impact on circuit response. By using Orcad's powerful description ability for circuit behavior characteristics, the fault propagation behavior could be simulated with two functions of Orcad parameter sweep and AC analysis. Hard fault only accounts for a small part (two extreme points) on the element variation range; as a result, it would be regarded as a special case of soft fault in the simulation process. By the simulation with node voltage as test parameters, we can obtain many groups of frequency -response curve cluster under the simulated fault states. The curves are plotted with response amplitude as ordinate against test frequency as abscissa. The waveform data will be save and convert to Matlab data files, such as *.mat. Through the Orcad and Matlab data communication interface program compiled by ourselves, we could further process and utilize the fault feature sample data.

\section{Creation of Node}

In this paper, the node set of the proposed fault propagation model is defined as : $E=(A, B)$, in which $\mathrm{A}$ is the fault pattern set according to the matching degree, and $\mathrm{B}$ is a Numerical constraints set of A, namely the information control points set $\mathrm{u}$ according to each fault pattern. For an analog circuit with $\mathrm{n}$ fault element and $\mathrm{m}$ fault node, if single fault is considered only, the element number of $\mathrm{E}$ is given by

$$
N=(n+m) \times \frac{1+\Delta}{\Delta}
$$


And if multi-fault is taken into account, the one is

$$
N=\left(\sum_{i} C_{n}^{i}+n+m\right) \times \frac{1+\Delta}{\Delta}
$$

in which $i$ is multiplicity of fault, $n \geq i \geq 2$ and $C_{n}^{i}$ is combinatorial operations.

\section{Creation of Arc}

After the sample points fuzzy partition of fault data, the simulation data is fallen under different pattern. For the convenience of numerical treatment, the matrix $Q_{h}=\left[Q_{h j}\right]$ is defined which describe the sample data distribution characteristics in each pattern. The $h$ is label of pattern, $j$ is label of sample data, and $Q_{h j}$ is the partition information content for sample $\mathrm{j}$ to fault pattern $\mathrm{h}$.. By comparison of different $Q_{h j}$, the matrix $R=\left[R_{k l}\right]_{K \times L}$ can be obtained which describe their coupling relationship. $\mathrm{K}$ and $\mathrm{L}$ are the pattern number of different circuit node, and $R_{k l}$ is the coupling information content between pattern $\mathrm{k}$ and pattern 1 , which can be shown as (3). $Q_{k}$ and $Q_{l}$ are the distribution characteristics matrix of pattern $\mathrm{k}$ and patter 1 , and SUM is summation operation.

$$
\left\{\begin{array}{c}
R_{k l}^{\prime}=\operatorname{SUM}\left(Q_{1} \bullet \times\left(Q_{k} \& Q_{1}\right)\right) \\
R_{k l}=R_{k l}^{\prime} / \sum R_{k l}^{\prime}
\end{array}\right.
$$

The matrix $R$ establishes the directed and weighted path of different circuit node which includes information in two aspects: Fault propagation direction $D(k \rightarrow l)$ and the weighted value of path $R_{k l}$ which describes the information distribution estimation of fault sample data and could be update through improving the statistical data. Thus the arc set of the proposed fault propagation model can be defined as $F=\left(D(k \rightarrow l), R_{k l}\right)$.

Till then we construct the analog circuit fault propagation network model, which can be described by

$$
G=(E, F)=\left((A, B),\left(D(\mathrm{k} \rightarrow l), \quad R_{\mathrm{kl}}\right)\right)
$$

\section{ANAlysis TOGETHER WITH A CASE STUDY}

Taken an international standards circuit under test shown as Fig. 2 as an example.

The nominal parametric values are

$$
\begin{aligned}
& R 1=10 K \Omega, R 2=12 K \Omega, R 3=11 K \Omega, R 4=9.5 K \Omega, R 5=8 K \Omega, R 6=6 K \Omega, \\
& R 7=9 K \Omega, R 8=6 K \Omega, R 9=10 K \Omega, R 10=10 K \Omega, R 11=10 K \Omega, R 12=10.5 K \Omega, \\
& C 1=0.01 \mu F, C 2=0.02 \mu F, C 3=0.02 \mu F, C 4=0.01 \mu F .
\end{aligned}
$$

With Pspice program, each element parameter and state variation can be simulated through AC Analysis and Parameter Sweep. Each circuit node frequency response under normal condition can be obtained as Fig. 3. And Fig. 4 is Node 4 frequency response under $\mathrm{R} 9$ fault.

According to the modeling steps, the fault propagation model with complex network and its parameter can be shown as Table I.

From Table I, we can find out that the circuit fault propagation model has big clustering coefficient and small average path length, and it can be considered that the model has small-world property, which has promoting effect on the fault propagation and spread. The model's degree distribution presents scale free feature which indicates the fault propagation information concentrates on a few network nodes. The node which has relatively large degree, especially out-degree, is corresponding to more propagation paths. So we could obtain optimal fault test node set from shortest path analysis which could guarantee accuracy of fault identification.

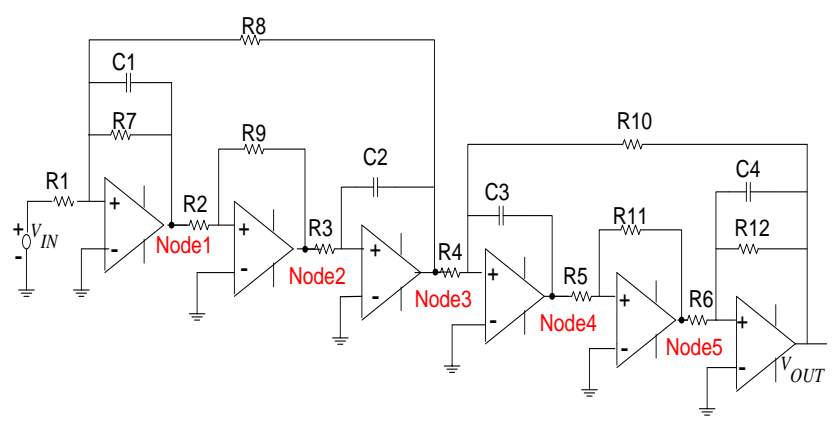

Fig. 2. Circuit instance.

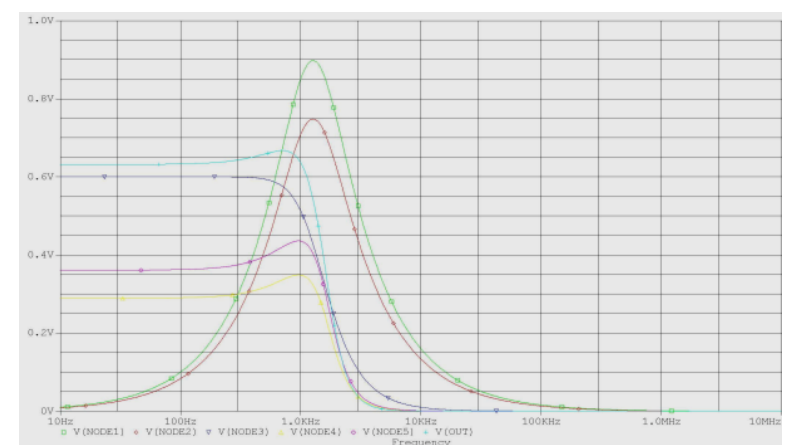

Fig. 3. Circuit node frequency response under normal condition.

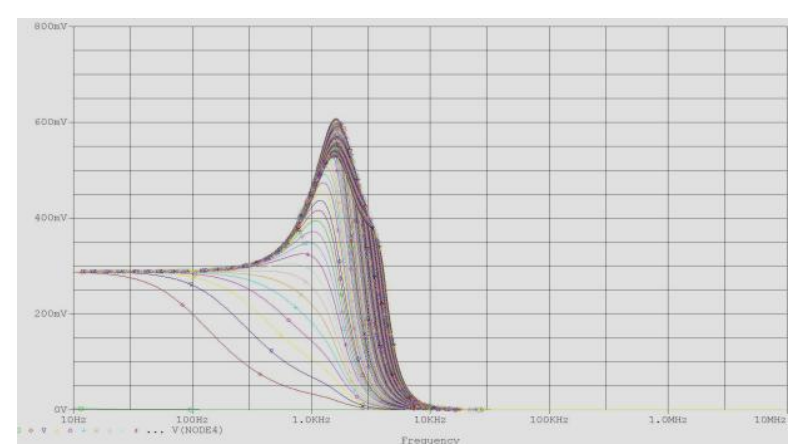

Fig. 4. Node 4 frequency response under R9 faul.

TABLE I: MODEL PARAMETERS

\begin{tabular}{lccccccc}
\hline & $N$ & $\sum k$ & $k$ & $\bar{d}$ & $c$ & $\gamma$ \\
\hline Instance model & 231 & 2318 & 10.03 & 2.74 & 0.15 & 1.5 \\
Radom graph & 231 & - & 10.03 & 2.36 & 0.04 & - \\
\hline
\end{tabular}

\section{CONCLUSION}

The proposed modeling and simulation method for analog circuit propagation behavior in this paper is an explorative and active study on the solution of information insufficiency in the analog circuit fault diagnosis and low robustness in fault identification and location. At last but not the least, together with a case study, the model proved to be efficient in 
providing the deep diagnosis knowledge. The method, which combines complex network with graph theory, can effectively solve resolution problem between soft-fault and tolerance and also he coupling relationship between fault patterns is not limited to linear case. Furthermore, from the parameter of the model, we can obtain the inherent mechanism of fault propagation in analog circuits including small world and scare-free properties, acquire same reference information for optimal selection of test points and reliability design.

\section{REFERENCES}

[1] M. F. Peng, J. J. Wang, C. K. Tse, and M. E. Shen, "Complex network application in fault diagnosis of analog circuit," International Journal of Bifurcation and Chaos, vol. 21, no. 5, pp. 1323-1330, 2011.

[2] H. Tan and M. F. Peng, "Minimization of ambiguity in parametric fault diagnosis of analog circuits: A complex network approach," Applied Mathematics and Computation, vol. 219, pp. 408-415, 2012

[3] M. F. Peng, M. E Shen, J. B. He, H. Xie, and Y. G. He, "Soft-fault detection and location for circuits with tolerance," Transactions of China Electrotechnical Society, vol. 24, no. 3, pp. 222-228, 2009

[4] M. F. Peng, Y. G. He, Y. N. Wang, and J. B. He, "Synthetic intelligence based fault diagnosis of analog circuits," in Proc. the CSEE, vol. 26, no. 3, pp. 19-24, 2006.

[5] P. Wang and S. Y. Yang, "A new diagnosis approach for handling tolerance in analog and mixed-signal circuits by using fuzzy math," IEEE Transactions on Circuits and Systems I: Regular Papers, vol 52, issue 10, pp. $2118-2127,2005$.

[6] P. Wang and S. Y. Yang, "Soft fault test and diagnosis for analog circuits," IEEE International Symposium on Circuits and Systems, vol. 3 , pp. $2188-2191,2005$.

[7] D. J. Watts and S. H. Strogatz, "Collective dynamics of 'small-world' networks," Nature, vol. 6, no. 393, pp. 440-442, 1998.

[8] S. H. Strogatz, "Exploring complex networks," Nature, vol. 3, no. 410, pp. 268-277, 2001.

[9] R. Albert and A. L. Barabási, "Statistical mechanics of complex networks," Rev. Mod. Phys, vol. 1, no. 74, pp. 47-97, 2002.

[10] B. Bollobás and O. Riordan, "Mathematical result on scale-free random graph," in Handbook of Graphs and Networks: From the Genome to the Internet, eds. S. Bornholdt, H. G. Schuster, Berlin: Wiley-VCH, 2003, pp. 1-34.

[11] R. Cohen and S. Havlin, "Scale-free networks are ultrasmall," Phys. Rev. Lett. , 90058701, 2003.

[12] A. Fronczak, P. Fronczak, and J. A. Holyst, "Meal-field theory for clustering coefficients in Barabási-Albert networks," Phys. Rev. E, vol. 68, pp. 046-126, 2003.

[13] M. E. J. Newman, "The Structure and Function of Complex Networks," Society for Industrial and Applied Mathematics, vol. 45, no. 2, pp. 167-256, 2003.

[14] X. F. Wang and G. Chen, "Complex networks: Small-world, scale-free and beyond," IEEE circuits and systems magazine, vol. 3, no. 2, pp. 6-20, 2003.

[15] J. H. Lu and G. R. Chen, "Analysis, control and applications of complex networks: A brief overview," in Proc. IEEE International Symposium on Circuits and Systems, pp. 1601-1604, 2009.

[16] W. M. Tam, C. M. Francis, and C. K. Tse, "Complex-Network Modeling of a Call Network," IEEE Circuit and System Society, vol. 56, no. 2 , pp. 416-429, 2009.

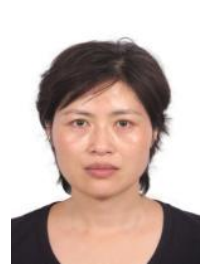

processing.
Meie Shen was born in Jiangsu, China, in 1966. She received the Ms. degree in 1989 in 2006, in automation from Huazhong University of Science and Technology, China.

She works as an associate professor in the Beijing University of Information Science and Technology. Her fields of interest are in test and diagnosis of analog circuits, circuit simulation and intelligent information

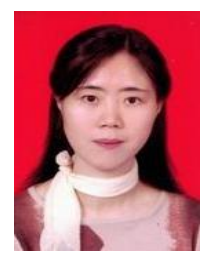

Minfang Peng was born in Hunan, China, in 1964. She received the Ms. degree in 1992 and $\mathrm{Ph}$. D. degree in 2006, both in electrician theory from Hunan University, China.

She works as a professor at the college of electrical and information engineering in Hunan University. Her fields of interest are in test and diagnosis of analog information processing. circuits, fault diagnosis of power system, intelligent 